\title{
Simple Method for Phosphorus Diffusion on <100> Oriented P-Type Silicon Using New Phosphorus Gel as Dopant
}

\author{
D. Kobor ${ }^{1}$, R. Ndioukane ${ }^{1}$, O. Palais ${ }^{2}$, M. Touré ${ }^{1}$, A. K. Diallo ${ }^{1}$, N. C. Y. Fall ${ }^{1} \&$ M. Biagui ${ }^{1}$ \\ ${ }^{1}$ Laboratoire de Chimie et de Physique des Matériaux (LCPM), Département de Physique, University Assane Seck \\ of Ziguinchor, Senegal \\ ${ }^{2}$ IM2NP (UMR CNRS 7334) - Aix-Marseille University, Case 231 - 13397 Marseille Cedex 20, France \\ Correspondence: Diouma Kobor, Laboratoire de Chimie et de Physique des Matériaux (LCPM), Département de \\ Physique, Université Assane Seck de Ziguinchor, Ziguinchor, Sénégal. E-mail: dkobor@univ-zig.sn
}

Received: January 14, 2014 Accepted: January 26, 2015 Online Published: February 20, 2015

doi:10.5539/apr.v7n2p49 URL: http://dx.doi.org/10.5539/apr.v7n2p49

\begin{abstract}
In this work, we try to make a p-type monocristalline silicon pn junction using an easier doping method. We combined spin-coating thin film deposition method and solid doping technique. This technique can be considered as variety of the SOD method.

In this study, phosphorous-based gel compounds was prepared and deposited by spin coating. Heat treatment would thus, after deposition of thin layer, diffuse phosphorus atoms into the substrate to obtain a pn diode. Study by Secondary Ions Mass Spectrometry (SIMS) showed a surface phosphorus concentration of $10^{20} \mathrm{at} / \mathrm{cm}^{3}$ incorporated within the silicon substrate to a depth of $300 \mathrm{~nm}$. The microwave phase-shift ( $\mu \mathrm{W}-\mathrm{PS}$ ) technique is used to determine the bulk lifetime $\left(\tau_{\mathrm{b}}\right)$ of minority carriers. In this technique, the phase-shift between a microwave beam $(10 \mathrm{GHz})$ and a sine-modulated infrared excitation is related to $\tau_{\mathrm{b}}$ and to the surface recombination velocity (S) (Palais, Clerc, Arcari, Stemmer \& Martinuzzi, 2003). The lifetime $\tau_{\mathrm{b}}$ mean values vary from $7 \mu$ s for a p-type Silicon to $97 \mu$ s for phosphorus-diffused silicon. The surface recombination velocity $\mathrm{S}$ varies from around 500 to $1000 \mathrm{~cm} \cdot \mathrm{s}^{-1}$.
\end{abstract}

Keywords: Monocristalline Silicon; phosphorus diffusion; Spin Coating; Lifetime, mapping.

\section{Introduction}

Silicon is a material that combines a set of mechanical, chemical, thermal, optical and electronic properties. All electronic and electrochemical properties make silicon material with great potential for the development of high technology. Nowadays, there are different products on the market of silicon high technology. However, further efforts to research and development have to be undertaken on the material and the technology implementation. Silicon required to manufacture components has reached a level of maturity to consider the realization of simple components. For photovoltaic or electronic applications, doping is needed to increase the silicon material properties. In this material, the member of the column IV of the periodic table, donors are the elements of column V (the most used are the phosphorus and arsenic), while the acceptors are the elements of the column III (Boron is most used). The electrical behavior of a semiconductor component such as silicon, strongly, depends to the dopants distribution within the structure. Several techniques were used to diffuse phosphorus in silicon for solar cells or electronic applications. But all of these techniques required expensive equipment. So it is necessary to find the lowest and easiest method to fabricate solar cells particularly. The combination of the film deposition and the solid dopant diffusion method can be used for phosphorus doping.

It exists many ways of preparing thin films that can be classified into physical and chemical pathways. The best methods known regarding the physical pathways are sputtering (Leprince-Wang, \& Yu-Zhang, 2001) and thermal evaporation (Takeda, Suzuki, Odaka, \& Hosono, 2001). These methods require expensive equipment. The privileged technical, in our case, regarding the chemical process is the "sol-gel" method (Ha, Nam, Lim, Oh, \& Hong, 1996).

In this paper, we propose a new method of doping using sol-gel method via two main steps: depositing a layer by spin coating and diffusion by thermal annealing. The originality of this method is the use of free organic gel obtained with the direct use of $\mathrm{P}_{2} \mathrm{O}_{5}$ oxide powder as the gel precursor. 


\section{Experimental procedure}

A boron doped p-type (100) silicon wafer of $600-625 \mu \mathrm{m}$ of thickness with resistivity of $1-5 \Omega . c m$ of the polished face was purchased from BT Electronics. The samples were cleaned by standard Radio Corporation of America (RCA) method to remove oxide followed by a 20-sec dip in 10:1 $\mathrm{H}_{2} \mathrm{O}: \mathrm{HF}$. After de-ionized water rinse and $\mathrm{N}_{2}$ blow was used to dry samples..

\subsection{Dopant Gel Synthesis and Thin Layer Deposition}

5 - $10 \mathrm{~g}$ of $98 \%$ extra pure Phosphorus pentoxide (white $\mathrm{P}_{2} \mathrm{O}_{5}$ ) from Acros/Fisher Scientific were weighed and slowly dissolved in $5-10 \mathrm{ml}$ of distilled water. The resulting solution is placed on a magnetic stirrer at low speed for 4 hours at temperature between 40 and $60{ }^{\circ} \mathrm{C}$. Thus, a yellow-brown dense gel (Figure 1) is obtained and ready to be deposited on the p-type Silicon sample. To obtain a homogeneous layer, spinning process was carried out at room temperature using a spin coater Midas $1200 \mathrm{D}$ at $3500 \mathrm{rpm}$ with an initial acceleration of 5 seconds and an operating time of $10 \mathrm{~min}$. After $10 \mathrm{~min}$ bake in oven at $100{ }^{\circ} \mathrm{C}$, thermal annealing in a $\mathrm{K} 114$ type muffle furnace was performed. The heating rate of the furnace is chosen between 10 and $20^{\circ} \mathrm{C} / \mathrm{min}$. A $30-60 \mathrm{~min}$ plateau was carried at $900{ }^{\circ} \mathrm{C}$ for the phosphorus diffusion through the sample. Cooling at ambient temperature was done naturally. After phosphorus diffusion, the sample was cleaned using HF solution for $30 \mathrm{~s}$ to remove the formed phosphosilicate glass. Deionized water was used to rinse the sample before characterizations. Phosphorus was diffused on polished $\langle 100\rangle$ oriented p-type monocristalline silicon face of 4 samples (DKRM-1, DKWB-1, DKWB-2 and DKNF) in same conditions at different dates for the diffusion method reproducibility.

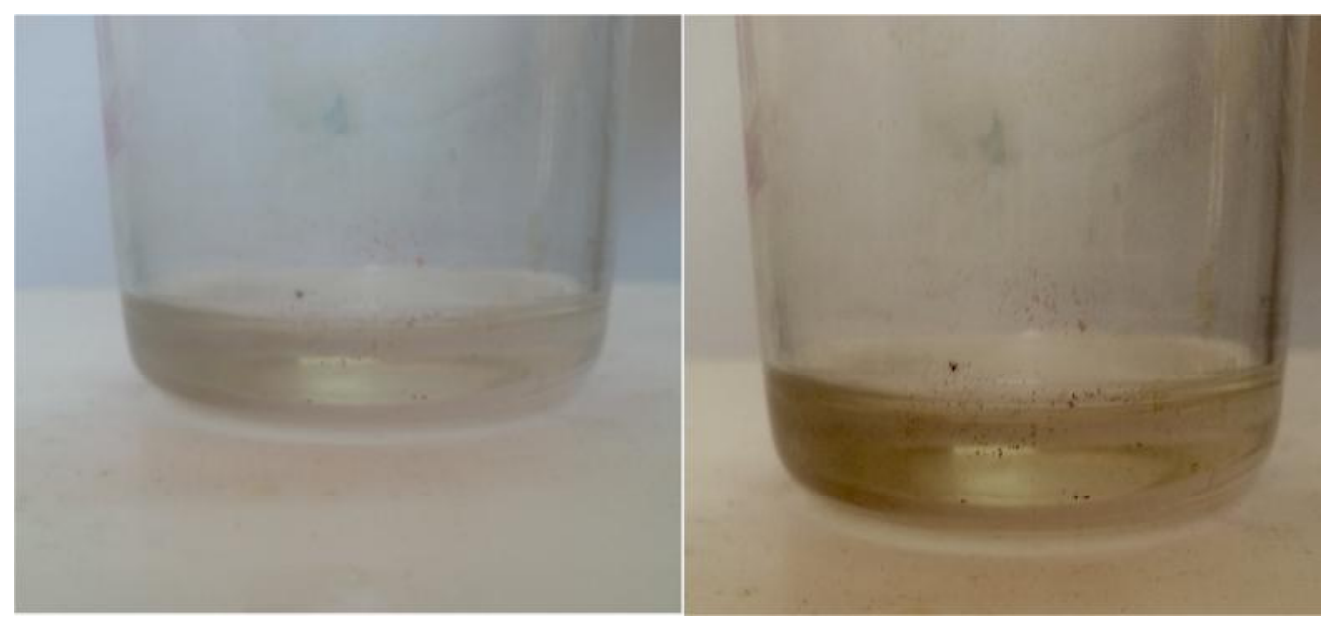

Figure 1. Free organic dopant gel fabricated from phosphorus oxide precursor

\subsection{Surface Morphology by SEM Observations}

The scanning electron microscopy and analytical techniques associated prove to be indispensable and unavoidable for the microstructural characterization of nanostructures obtained tools. They provide structural, morphological and chemical information on individual nanoscale grains simultaneously. Thus they can consolidate, understand and validate other analytical results obtained by other characterization techniques in order to establish the relationship between microstructures and properties. Surface morphology of phosphorus doped silicon samples were observed by using a MERLIN FEG of Zeiss scanning electron microscopy model in ICMPE (Paris-Est University in France).

\subsection{SIMS Analysis}

The SIMS measurements were carried out using an IMS 7f N (CAMECA) last generation model. All analysis were performed in primary $\mathrm{C}_{\mathrm{s}}^{+}$/negative secondary high mass resolution to remove ${ }^{31} \mathrm{P}^{30} \mathrm{Si}^{1} \mathrm{H}$ interference $(\mathrm{M} / \Delta \mathrm{M})=10000$. The size of the spray crater is about $(150 \mathrm{X} 150) \mu \mathrm{m}^{2}$. DEKTAK 8 profilometry was used to determine the depth of sputtered craters and calibrate the depth scale assuming a constant sputter rate for the entire profile. Concentrations of $\mathrm{P}$ in the $\mathrm{Si}$ were calculated using a relative sensitivity factor determined from a standard sample. 


\subsection{Carriers Lifetime and Recombination Velocity Mappings}

The microwave phase-shift ( $\mu$ W-PS) technique is used to determine the bulk lifetime $\left(\tau_{\mathrm{b}}\right)$ of minority carriers. In this contactless technique, the phase-shift between a microwave beam $(10 \mathrm{GHz})$ and a sine-modulated infrared excitation is related to $\tau_{\mathrm{b}}$ and to the surface recombination velocity (S). With $\mu \mathrm{W}$-PS, a harmonic modulated laser light excites the sample so that the injection level is quasi constant during all the measurements, thereby attenuating the problems connected to the surfaces. The phase shift between the modulation and the microwave reflected by the sample is measured. From this phase shift, the bulk lifetime as well as the surface recombination velocity of the illuminated side (front side), $\mathrm{S}$, can be deduced by several measurements at different modulation frequencies (Palais, Clerc, Arcari, Stemmer \& Martinuzzi, 2003). In this study, we used the sample DKRM-1 for its phosphorus diffusion depth in the silicon and its perfect SIMS profile and reproducibility.

\subsection{Electrical Measurement: Sheet Resistance Determination}

With the lifetime of charges carriers, the resistivity $(\rho)$ is an important characteristic for a semiconductor material. Indeed, resistivity measurement is used to know the initial doping value $\left(\mathrm{N}_{\mathrm{A}}\right.$ for the p-type silicon and $\mathrm{N}_{\mathrm{D}}$ for silicon n-type) of an uncompensated silicon sample. In the case of p-type silicon $\rho=1 /\left(q p \mu_{p}\right)$ with $p \approx N_{A}$ and in the case of n-type silicon $\rho=1 /\left(\mathrm{qn} \mu_{\mathrm{n}}\right)$ with $\mathrm{n} \approx \mathrm{N}_{\mathrm{D}}$, q representing the electron elementary charge, and $\mu_{\mathrm{n}}$ and $\mu_{\mathrm{P}}$, respectively the electrons and holes motilities, measuring resistivity of a semiconductor sample is carried out using four equidistant tungsten points (spacing e) (Microworld, 2014). For this measure to be valid it is necessary that the surface of the measured layer is considered to be infinite $(>10 \mathrm{e})$, sufficiently thin $(<0.4 \mathrm{e})$ and the measurement is performed at $25^{\circ} \mathrm{C}$. It is then possible to determine the resistivity of the layer:

$$
\rho=R_{\square} x d
$$

$\mathrm{d}$ is the thickness of the measured layer. The measurements were done on DKRM-1 sample with the same reasons explained for the minority carrier lifetime mapping.

\section{Results and Discussions}

\subsection{SEM Images Observations}

Figures $2 \mathrm{a}$ and $2 \mathrm{~b}$ show silicon surface after phosphorus diffusion. It has little sized patches with deep penetration inside the material. In Figures $2 \mathrm{c}$ and $2 \mathrm{~d}$ more large sized patches on the surface of the wafer were found with also deep penetration. The presence of white nanoparticles on the surface can be explained by the phosphosilicate glasses (PSG) formation increasing the surface reflectance of the Figure $2 \mathrm{c}$ and Figure $2 \mathrm{~d}$ images compared to Figure $2 \mathrm{a}$ and Figure $2 \mathrm{~b}$ ones. They could be due to the phosphorus oxide germination. Indeed, in presence of oxygen, phosphosilicate glass (PSG) is formed at the $850{ }^{\circ} \mathrm{C}$ temperature. Phosphosilicate glass or PSG is phosphorus doped silicon dioxide, a hard material formed at the top surface of Si wafer.

\subsection{SIMS Analysis}

Figure 3 shows depth profiling of $\mathrm{P}$ atoms observed by (SIMS) into the different samples. It can be seen from Figure 3a to Figure 3e that samples show reproducible presence of phosphorus diffused into the silicon. The ability to choose the study area reinforces the impression that the result is not related to an analysis "artifact" such as the presence of a residue deposition in the study area. The diffusion depth of the phosphorous atoms varies from 300 to $400 \mathrm{~nm}$ for DKRM-1 (Figure 3a, 3b, 3c and 3d) and from 175 to $250 \mathrm{~nm}$ for DKWB1 (Figure 3e) while it is equal to $350 \mathrm{~nm}$ for DKNF (Figure 3f). The dopant concentration value is the same for DKRM-1 and DKWB1 decreasing from $10^{20}$ at. $\mathrm{cm}^{-3}$ to around $10^{17} \mathrm{at}_{\mathrm{cm}} \mathrm{cm}^{-3}$ from the front surface to the diffusion limit with a perfect reproducibility of the SIMS analysis. They show the diffusion homogeneity around the samples surface. This behavior can be confirmed by the SEM images, which show perfect distribution homogeneity of observed patches before PSG removing. The phosphorus initial concentration $\left(2.10^{19}\right.$ at. $\left.\mathrm{cm}^{-3}\right)$ in DKNF is slightly less important than that found in DKRM-1 and DKWB1. This can be explained by the dopant thin layer thickness, which can change slightly depending to the gel density. These results confirm that the phosphorus can be incorporate inside the silicon with our sample method with a high injection as published with other more expensive diffusion methods. The diffusion depth is comparable to those from classic phosphorus diffusion methods in the literature (Biro et al., 2002) (see Table 1). 
Table 1. The phosphorus doped surface physic-chemical properties

\begin{tabular}{llllll}
\hline Samples & {$[\mathrm{P}]_{0}\left(\mathrm{~cm}^{-3}\right)$} & $\mathrm{b}(\mu \mathrm{s})$ & $\mathrm{S}\left(\mathrm{cm}^{-1} \mathrm{~s}^{-1}\right)$ & $\mathrm{R}_{\square}(\quad / \mathrm{sq})$ & Diffusion depth $(\mathrm{nm})$ \\
\hline$<100>$ p-type mc-Si & - & $6-7$ & - & 252 & - \\
DKRM-1 & $\approx 1 \times 10^{20}$ & $70-100$ & $570-1000$ & 22 & $300-400$ \\
DKWB-1 & $\approx 2 \times 10^{19}$ & - & - & - & $175-250$ \\
DKNF & $\approx 1 \times 10^{20}$ & - & - & - & $\approx 350$ \\
& & & & & $300-350$ SOD \\
[5] (After P diffusion) & $7 \times 10^{20}$ & $10-100$ & $250-1000$ & $20-60$ & $200-250$ APCVD \\
\hline
\end{tabular}
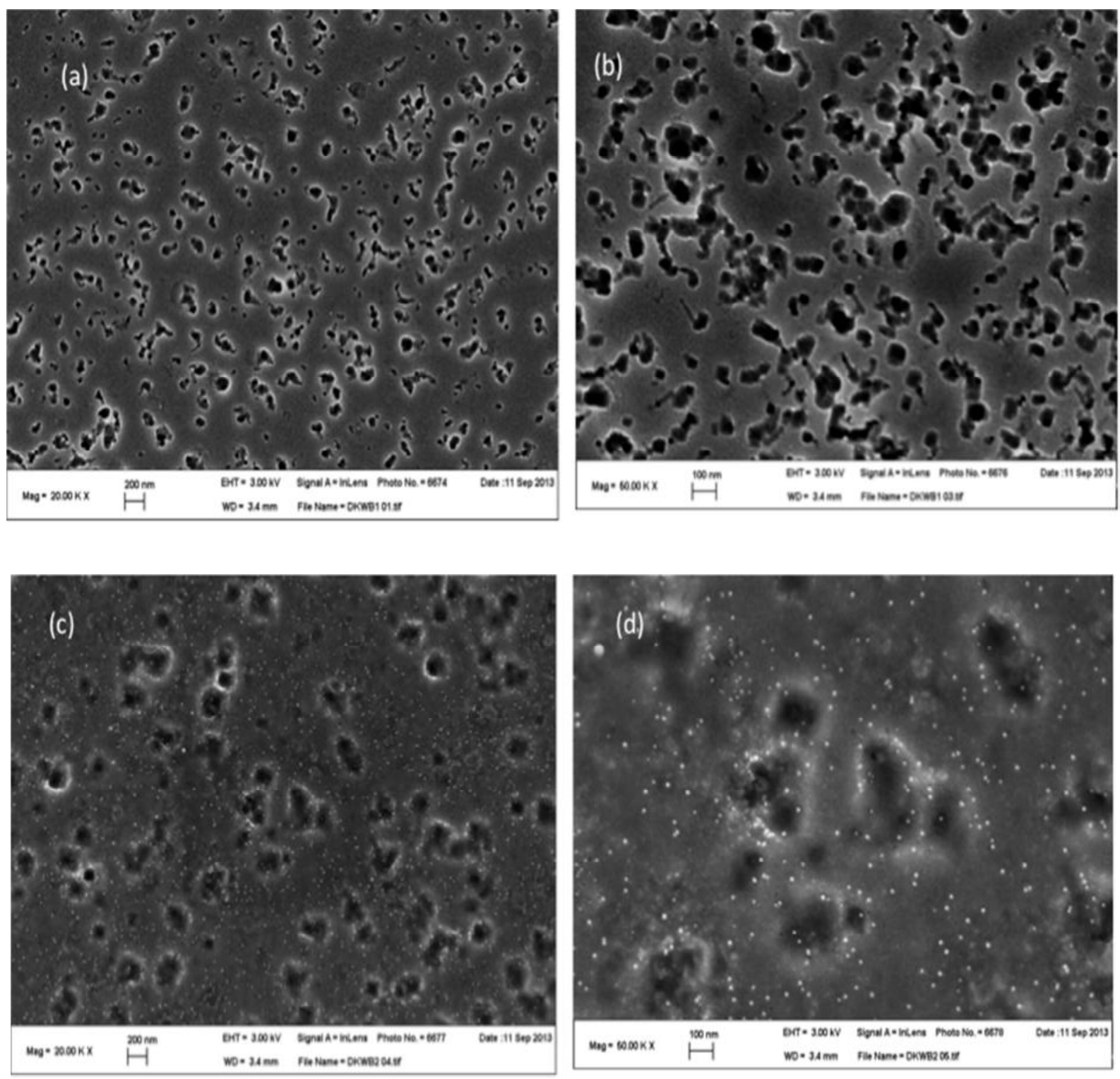

Figure 2. Scanning Electronic Microscopy images of DKWB1 with (a) $20 \mathrm{kX}$ (b) $50 \mathrm{kX}$ and of DKWB2 with (c) $20 \mathrm{kX}$ and (d) $50 \mathrm{kX}$ before PSG removing 

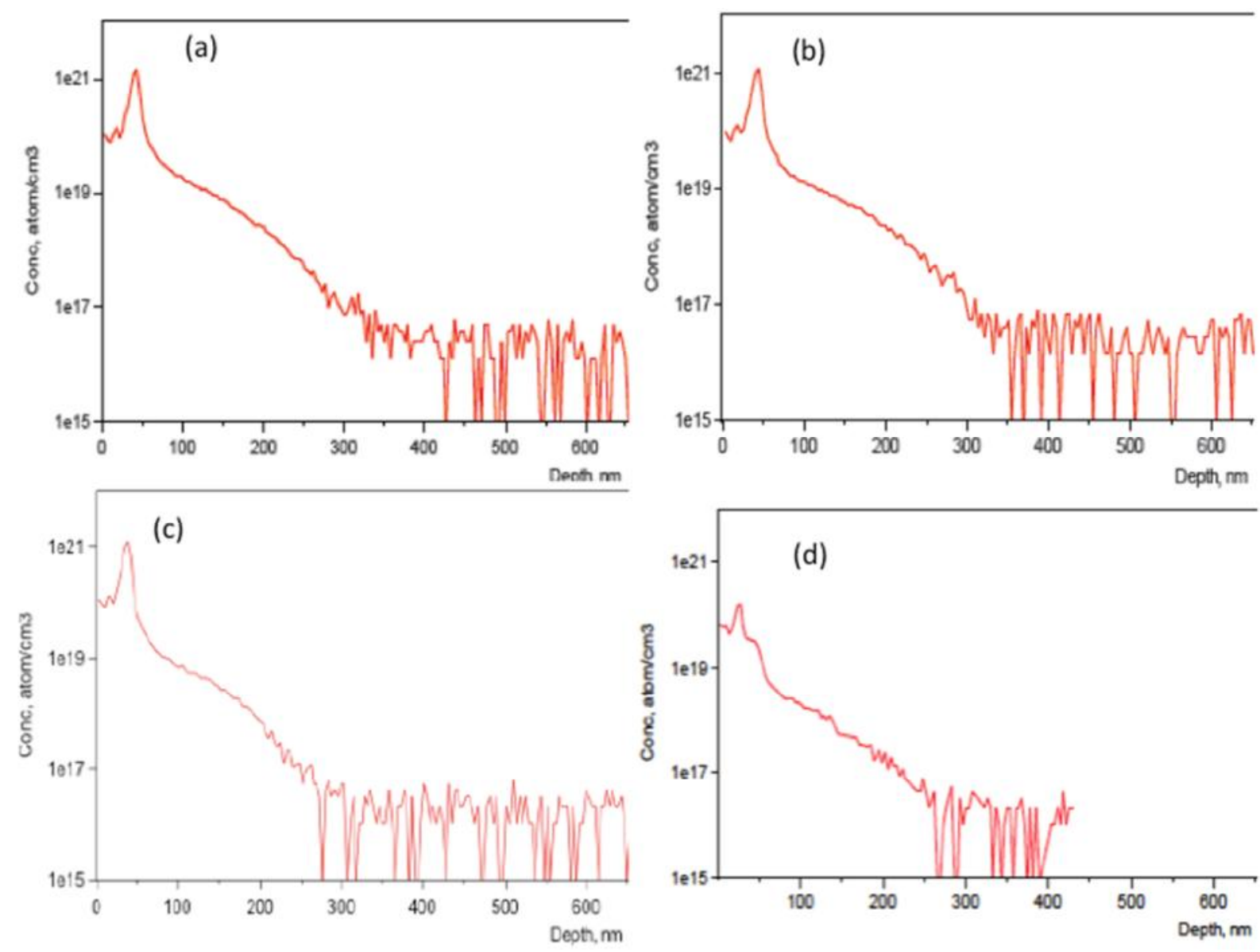

(e)
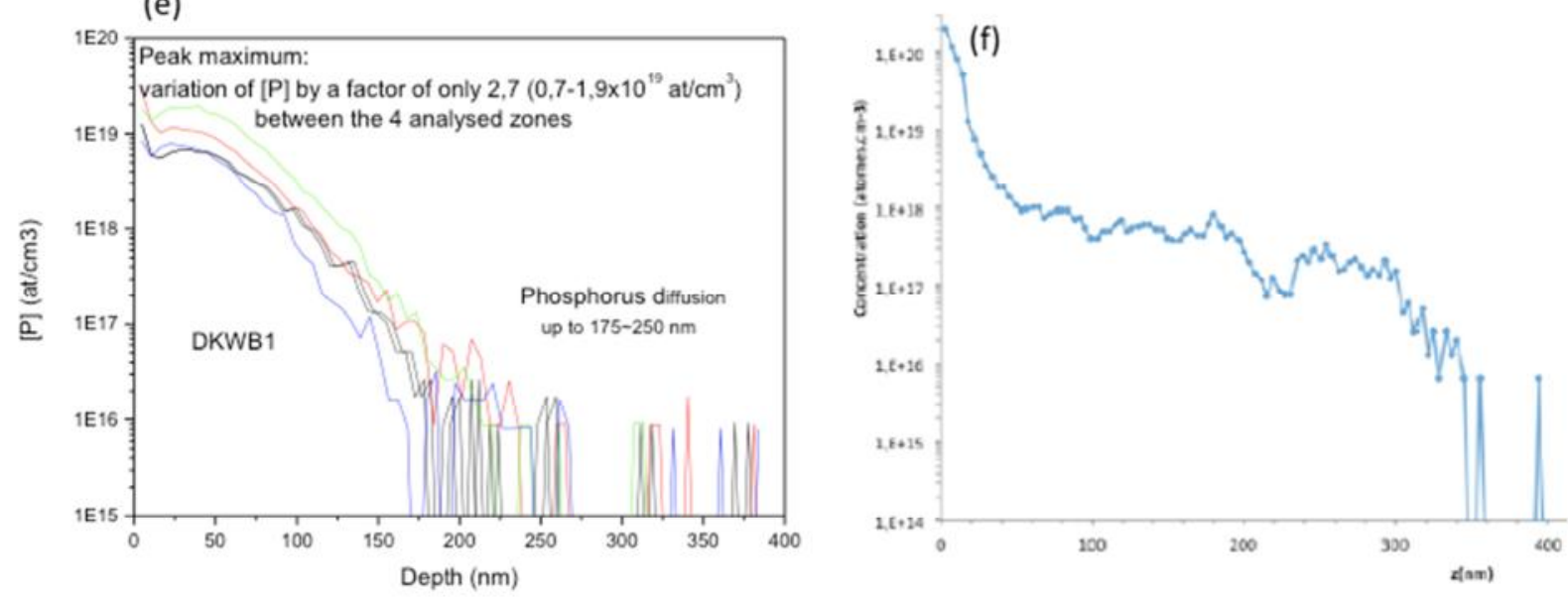

Figure 3. The Phosphorus ${ }^{31} \mathrm{P}$ concentration variation versus the substrate depth for 3 samples DKRM-1((a), (b) (c) and (d)), DKWB1 (e) and DKNF (f) doped using the new doping method by SIMS analysis for reproducibility test

\subsection{Carriers Lifetime and Recombination Velocity Mappings}

Figure 4 shows the minority carriers bulk lifetime for DKRM-1 sample before phosphorus diffusion (Figure 4a) and after phosphorus diffusion of p-type monocristalline silicon using our new method (Figure 4b). A significant improvement in bulk lifetime, from $\sim 6-7 \mu$ s to $\sim 70-100 \mu \mathrm{s}$, was observed after the phosphorus diffusion. This improvement reflects the increase in bulk as well as surface recombination lifetime during phosphorus diffusion. These results were found to be in very good agreement with the best results from literature (Iftiquar et al., 2012; Parola et al., 2014). In Figure 4b, we see two different areas: one on top of the mapping and the second 
on the bottom. The first area is polished with the chemical reagent CP4 while the second is a non-cleaned phosphorus diffused silicon surface. The CP4 use improved largely the minority carrier lifetime from around 90 to $100 \mu \mathrm{s}$. This improvement is attributed to the removal of contaminants and structural defects from the silicon surface after polishing and oxide removal.

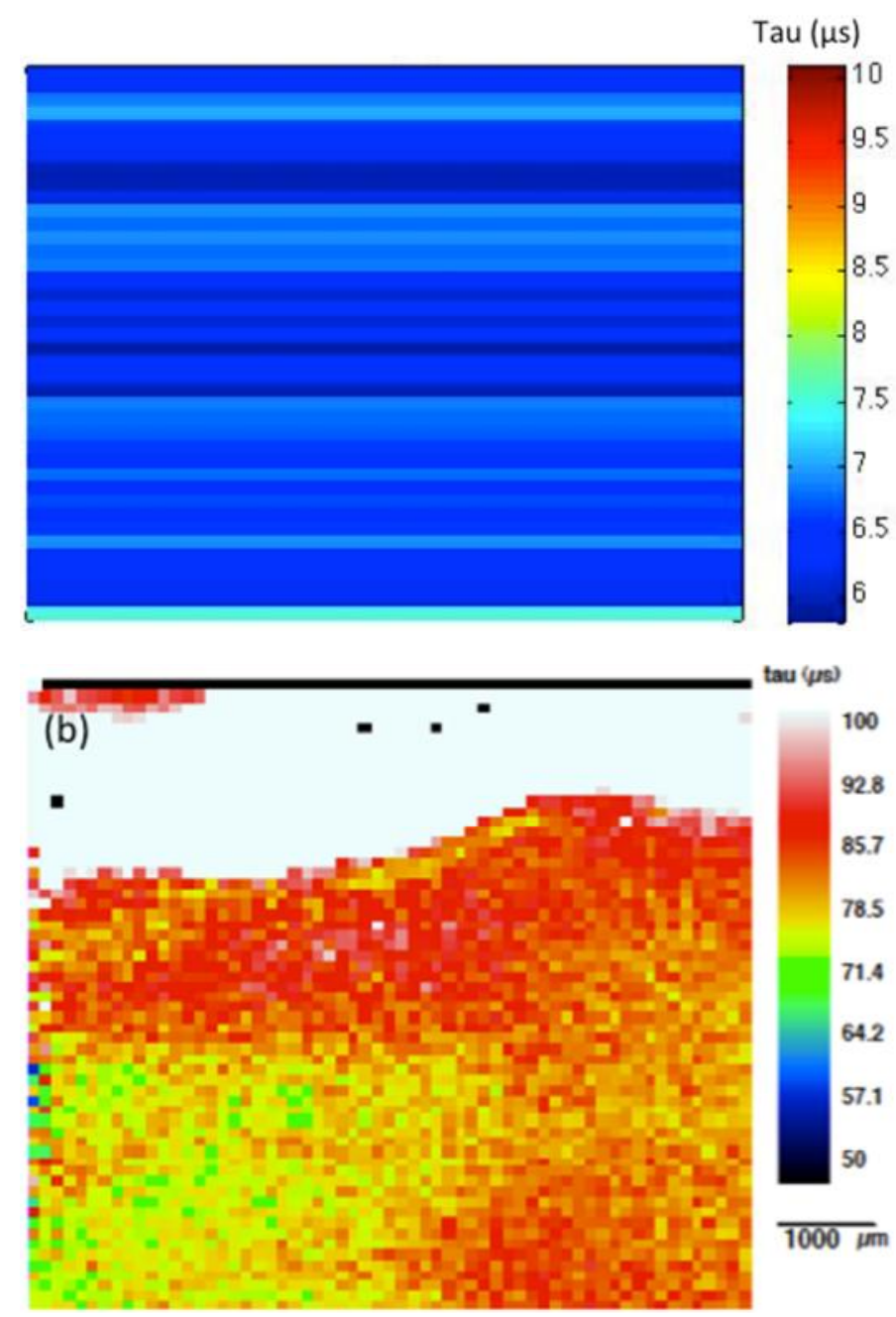

Figure 4. Carriers bulk lifetime $\tau_{\mathrm{b}}$ mapping of mono Si p-type surface (a) before phosphorus diffusion and (b) after single sided phosphorus diffusion for DKRM-1

The Figure 5 shows the phase shift and the minority carrier recombination velocity mapping of the each surfaces of the sample DKRM-1. From the phase shift (Figure 5a), the bulk lifetime as well as the surface recombination velocities of the illuminated side (front side) can be deduced by several measurements at different modulation frequencies. The phase-shift is related to parameters by following equation:

$$
\phi=f\left(\tau_{b}, S_{f}, S_{b}, D, W, \alpha, F\right)
$$

Among the seven parameters of Eq. (2), four are known: the measured phase-shift $(\Phi)$, the sample thickness (W), the absorption coefficient $(\alpha)$ and the modulation frequency $(F)$. To determine $n$ unknown parameters, the measurements must be repeated at $\mathrm{n}$ different frequencies (Palais, Clerc, Arcari, Stemmer \& Martinuzzi, 2003). This graph shows that the influence of the front surface on the recombination velocity is very strong. In Figure $5 \mathrm{~b}$, the first area of the surface shows a large recombination velocity of minority carrier $\mathrm{S}$ around $1000 \mathrm{~cm} . \mathrm{s}^{-1}$. It is more important than those for the second area, which vary from 500 to $850 \mathrm{~cm} . \mathrm{s}^{-1}$. These results could be considered as surprising but the improvement of the velocity in the polished area would be explained by that phosphorus diffusion created a passivation of the silicon surface decreasing the recombination rate while the cleaning with $\mathrm{CP} 4$ improves surface reactivity and so the recombination velocity. These results show that the 
minority carrier bulk lifetime is totally different from their surface recombination lifetime. In the case of high recombination velocity, this latter dominates the effective lifetime. The $\mathrm{S}$ is an indicator of surface passivation for silicon substrate (Iftiquar et al., 2012).
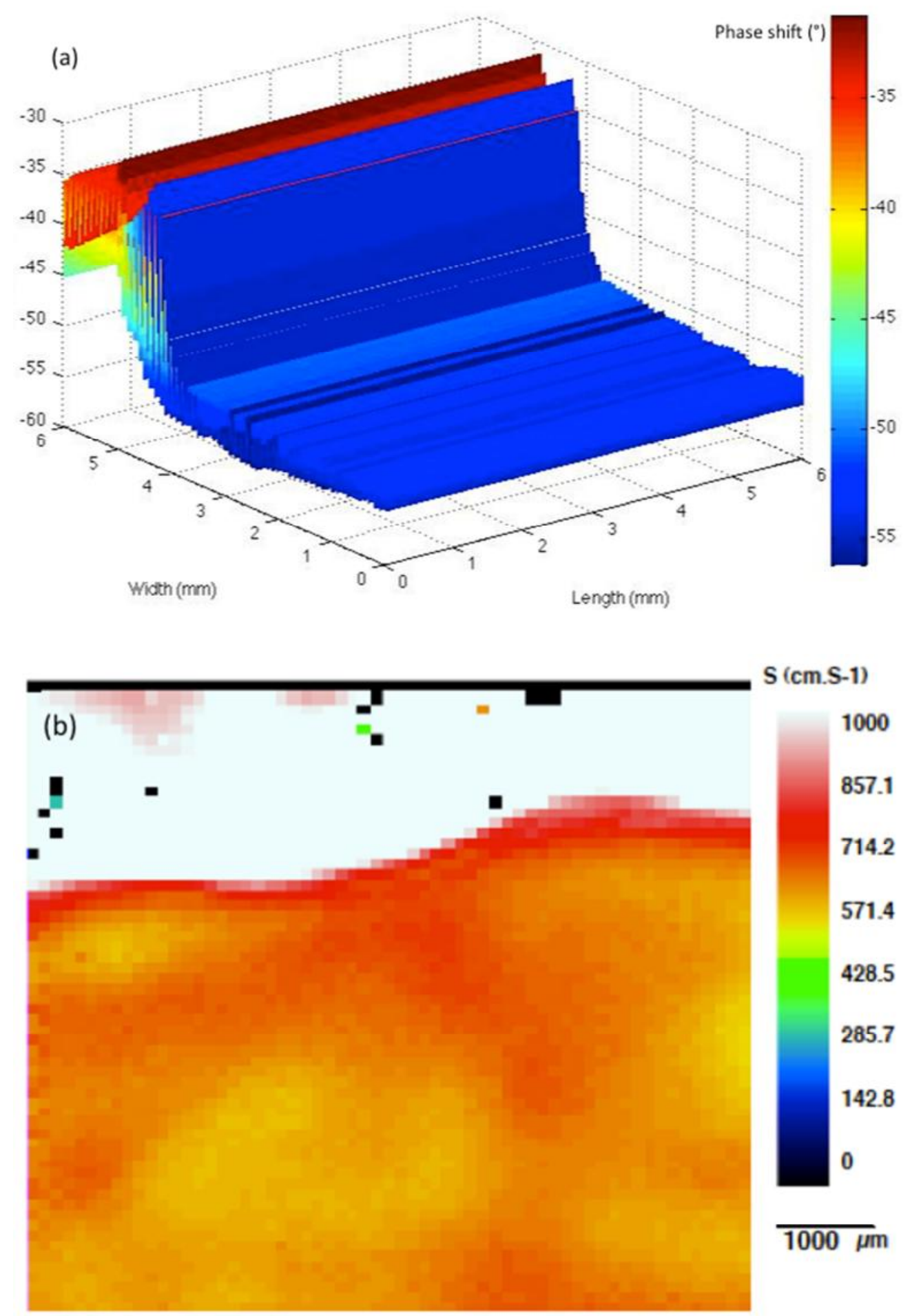

S (cm.S-1)
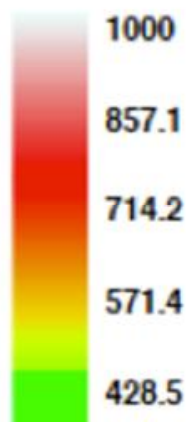

285.7

142.8

0

$\overline{1000 \mu \mathrm{m}}$

Figure 5. (a) Phase - shift and (b) Recombination velocity mappings for a sample after single sided phosphorus diffusion obtained by $\mu \mathrm{W}-\mathrm{PCD}$ for DKRM-1 


\subsection{Electrical Measurement}

Figure 6 shows the direct current versus the potential of the surface layer using the "four points" electrical method for the sample DKRM-1. The value of the resistance sheet decreases from $252 \Omega / s q$ for the p-type silicon to $20 \Omega / \mathrm{sq}$ after phosphorus diffusion on the sample surface. These values are in agreement with the literature (Iftiquar et al., 2012). For sheet resistance of 252 and $20 \Omega / s q$, the carrier mean lifetimes were around 6 and $97 \mu$ s respectively. We observed that, as the sheet resistance increases, the carrier lifetime decreases. This result shows that the sheet resistance measurement can provide important information on the phosphorus diffusion. The $\mathrm{n}$ doping creates an increase in carrier mobility so electrical conduction by the inclusion of electrons from donors. Indeed, relation between resistivity and carriers mobility and concentration for $\mathrm{n}$ type mono-Silicon

can be expressed as $\rho=\frac{1}{e \cdot n \cdot \mu_{n}}$ where e is the electron charge, $\mathrm{n}$, the dopant concentration and $\mu_{\mathrm{n}}$ the carrier

(electron) mobility. It is clear that the phosphorus diffusion will create an increase of donors rate so electrons (as carriers) concentration $\mathrm{n}$. The donor's concentration increase is then related to the carrier's rate increase, which will improve, of course, the layer conductivity reducing in fact its resistivity. In addition, the importance value of the electrons mobility in silicon $\left(1350 \mathrm{~cm}^{2} / \mathrm{Vs}\right)$ compared to holes mobility in silicon substrate $\left(480 \mathrm{~cm}^{2} / \mathrm{Vs}\right)$ could explain this resistance decrease because the mobility will change from holes mobility to electrons mobility in the diffusion zone.

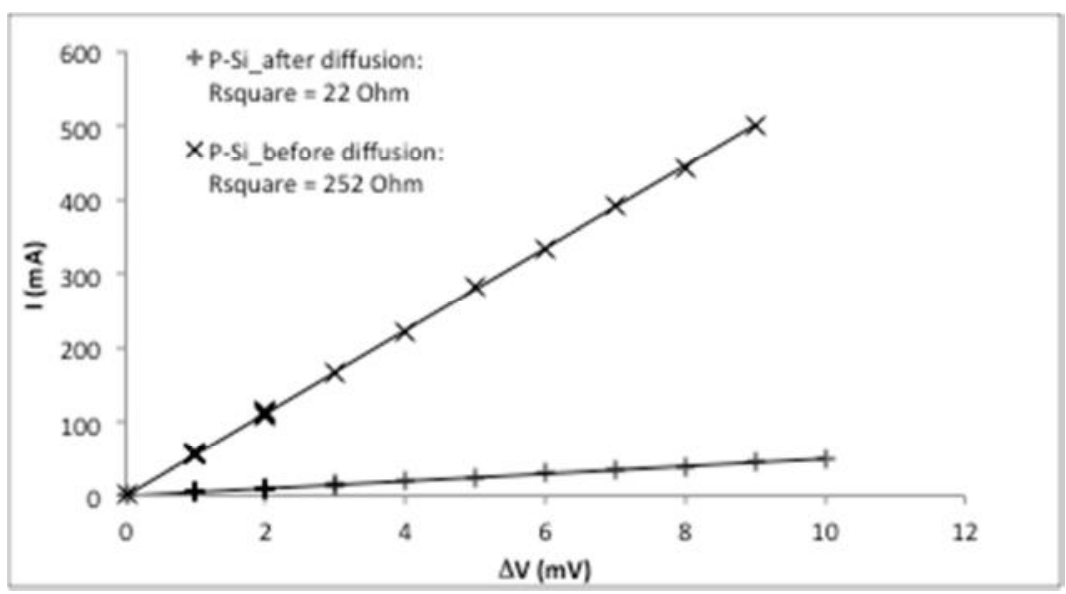

Figure 6. Current evolution versus voltage before and after single sided phosphorus diffusion for sheet resistance determination by 4-point method for DKRM-1

\section{Conclusion}

The SIMS and minority carrier lifetime results confirm that Phosphorus was well diffused within the silicon substrate using our new and simpler method. So we can say unequivocally that our objective was almost reached although. Results found and reproducibility of the technique in this work showed the possibility to realize very good doping using this simple method. The relation between minority carrier recombination velocity and their bulk lifetime needs strong study. Research must be performed to control the phosphorus diffused concentration acting on the thin layer thickness and the dopant gel density. Other investigations will be taken to use this method in silicon nanowires for solar cells application.

\section{Acknowledgments}

Financial support of the Senegalese Ministry of High Education and Research and the Economic Organization of West African States (ECOWAS) is gratefully acknowledged.

\section{References}

Biro, D., Preu, R., Schultz, O., Peters, S., Huljic, D. M., Zickermann, D., ... \& Willeke, G. (2002). Advanced diffusion system for low contamination in-line rapid thermal processing of silicon solar cells. Solar energy materials and solar cells, 74(1), 35-41. http://dx.doi.org/10.1016/S0927-0248(02)00045-4 
Ha, H. Y., Nam, S. W., Lim, T. H., Oh, I. H., \& Hong, S. A. (1996). Properties of the TiO 2 membranes prepared by CVD of titanium tetraisopropoxide. Journal of membrane science, 111(1), 81-92. http://dx.doi.org/10. 1016/0376-7388(95)00278-2

Iftiquar, S. M., Lee, Y., Ju, M., Balaji, N., Dhungel, S. K., \& Yi, J. S. (2012). Fabrication of Crystalline Silicon Solar Cell with Emitter Diffusion, SiNx Surface Passivation and Screen Printing of Electrode. http://dx.doi.org/10.5772/51065

Leprince-Wang, Y., \& Yu-Zhang, K. (2001). Study of the growth morphology of TiO 2 thin films by AFM and TEM. Surface and Coatings Technology, 140(2), 155-160. http://dx.doi.org/10.1016/S0257-8972(01)01029-5

Microworld. (2014). Four Point Probe Resistivity. Retrieved from www.four-point-probe.eu

Palais, O., Clerc, L., Arcari, A., Stemmer, M., \& Martinuzzi, S. (2003). Mapping of minority carrier lifetime and mobility in imperfect silicon wafers. Materials Science and Engineering: B, 102(1), 184-188. http://dx.doi.org/10.1016/S0921-5107(02)00652-9

Parola, S., Daanoune, M., Focsa, A., Semmache, B., Picard, E., Kaminski-Cachopo, A., \& Blanc-Pélissier, D. (2014). Study of photoluminescence decay by time-correlated single photon counting for the determination of the minority-carrier lifetime in silicon. Energy Procedia, 55, 121-127. http://dx.doi.org/10.1016/j.egypro. 2014.08.091

Takeda, S., Suzuki, S., Odaka, H., \& Hosono, H. (2001). Photocatalytic TiO 2 thin film deposited onto glass by DC magnetron sputtering. Thin Solid Films, 392(2), 338-344. http://dx.doi.org/10.1016/S0040-6090(01)01054-9

\section{Copyrights}

Copyright for this article is retained by the author(s), with first publication rights granted to the journal.

This is an open-access article distributed under the terms and conditions of the Creative Commons Attribution license (http://creativecommons.org/licenses/by/3.0/). 